\title{
INTELLIGENT NETWORKED HYBRID ELECTRIC VEHICLE OPTIMIZATION USING A LINEARIZED ECMS CONTROL ALGORITHM
}

\author{
Mengxiong Lu \\ Changzhou College of Information Technology, Changzhou, 213164, China \\ Email: lumengxiong06@126.com
}

\begin{abstract}
The purpose is to promote the development of the automotive industry, develop energysaving, safe, comfortable, and environment-friendly HEVs (Hybrid Electric Vehicle). Here, an HEV fleet is researched through the intelligent network connection to improve the fuel economy, traffic fluency, comfort, and safety of the vehicle. The HEV based on linearized ECMS (Equivalent Fuel Consumption Minimization Strategy) control algorithm is partially optimized. Based on the control algorithm of ECMS, cA-ECMS [Equivalent Factor Adaptive Control Strategy Based on Continuous SOC (System on Chip) Feedback] and dA-ECMS (Equivalent Factor Adaptive Control Strategy Based on Discrete SOC Feedback) are proposed. Based on SOC feedback, an online identification A-ECMS (Adaptive ECMS) algorithm is established. The results show that the control algorithm gives full play to the advantages of the adaptive control algorithm and improves the adaptability of the equivalent factor of the HEV. Besides, the fuel consumption, motor total energy consumption, and SOC end value deviation under different SOC initial values are compared. The results show that with the increase of SOC initial value, the end state SOC deviations of CA-ECMS and dA-ECMS control algorithm are about $0.6 \%$ and $-1.6 \%$, respectively, but the fuel consumption is increasing. Meanwhile, the cA-ECMS control algorithm tends to use fewer batteries. From the perspective of adaptability of equivalent factor, the equivalent factor of the dA-ECMS control algorithm changes less and has better adaptability. All of these lay a solid theoretical foundation for the establishment of an energy management control algorithm for real-time control of the real vehicle.
\end{abstract}

Keywords: ECMS Control Algorithm; Intelligent Network Connection; Hybrid Electric Vehicle; System Optimization.

\section{Introduction}

With the continuous growth of people's travel demand and logistics transportation, the automobile industry has developed rapidly, advancing economic growth. Concurrently, a series of problems have been raised, such as excessive consumption of oil resources, environmental pollution, and the greenhouse effect [1]. Over the past decade, China's automobile production and per capita auto ownership rate have increased dramatically, and oil consumption has increased year by year, causing more and more serious energy security problems in China. The emergence of hybrid technology alleviates the problem of huge oil consumption.

Moreover, hybrid technology is one of the effective methods to solve energy security problems, optimize energy use structure, and reduce automobile exhaust pollution. HEVs (Hybrid Electric Vehicles) can meet the power requirements, improve fuel economy, and reduce exhaust emissions. It is acknowledged globally that compared with pure electric vehicles, HEVs can avoid many problems, including insufficient battery endurance and frequent charging problems. Since the 1990s, countries have issued guidance documents on new energy technologies and set up funds to encourage research institutions and enterprises to invest in the development and promotion of HEVs.

Commonly used HEVs provide required torque from two energy sources. Different from the traditional fuel vehicles, the additional power source is faced with a challenging energy optimal allocation problem. The HEV energy management algorithm can calculate the minimum objective function value for the torque/power distribution between fuel and battery systems under an overall driving condition.

In general, the following factors should be considered in calculating the optimal value: fuel consumption, emission, and battery aging [2-4]. The ECMS (Equivalent Fuel Consumption Minimization Strategy) unifies instantaneous fuel consumption and power consumption into equivalent fuel consumption through the oil-electricity conversion coefficient (also called an equivalent factor) and solves the optimal control decision. ECMS is a 
research hotspot in the real-time application of energy management algorithms because its calculation is simple and fast and can meet the realtime requirement of online applications. Since the algorithm relies heavily on the oil-electricity conversion coefficient, for an optimization effect of the algorithm, the A-ECMS (Adaptive ECMS) is usually adopted in the current research. Some scholars have compared three A-ECMS strategies of SOC (System on Chip) feedback control, and the results have shown that a better instantaneous optimization effect can be obtained through PI (Proportional Integral) control of equivalent factor [5-7]. Some researchers have improved the ECMS algorithm by combining PMP (Pontryagin Minimum Principle) offline processing and ECMS online operation, considering the influence of SOC on the adaptive rate [8]. Other researchers of parallel HEVs have deduced the upper and lower limit of the equivalent factor according to the efficiency of the engine, battery, and motor components. Meanwhile, they have changed the equivalent factor value according to the current SOC state and its upper and lower limit [9]. The real vehicle test results have confirmed that the ECMS algorithm with known working conditions can achieve a better fuel-saving effect through the comparison between the influence of known driving conditions and unknown driving conditions on the ECMS algorithm [10].

The energy management algorithm based on ECMS or A-ECMS can achieve real-time optimal control, but the difficulty lies in the reasonable selection of equivalent factors. Current research focuses on the real-time correction of equivalent factors through working condition information, running state, and traffic information to optimize the algorithm and ensure its real-time performance.

\section{Control Algorithm of ECMS 2.1 PMP}

PMP is proposed by the Soviet scholar Pontryagin through the improvement of the classical variational method and is widely used for solving the optimization problem of control variable constraints [11]. PMP is essentially a necessary rather than sufficient condition for solving a set of optimal control. Generally, the minimum control law that satisfies the performance index function is called the extremum. The optimum must be the extremum, but the extremum is not necessarily optimum. If the solution of the optimal problem is determined according to the conditions in practical problems, and the extremum calculated by PMP is the only solution, the obtained control is the optimum [1214].

In the PMP algorithm, the boundary interval of the state variable $x(t)$ is chosen as $\Omega_{x}(t)$, that is, $x$ $(t) \in \Omega_{x}(t)$, and $\forall t \in\left[t_{0}, t_{f}\right]$. The boundary interval of the control variable $u(t)$ is denoted as $U(t)$, that is, $u$ $(t) \in U(t)$. The dynamic equation of the control system is defined as equation (1).

$$
\dot{x}(t)=f(x(t), u(t), t)
$$

Then, the cost function can be expressed as equation (2).

$$
J(u)=\int_{t_{0}}^{t_{T}} L[x(t), u(t), t] d t
$$

In equation (2), $L[x(t), u(t), t]$ represents the instantaneous cost.

The covariate is set as in equation (3).

$$
\lambda^{T}(t)=\left[\lambda_{1}(t), \lambda_{2}(t), \lambda_{3}(t), \ldots, \lambda_{n}(t)\right]
$$

In equation (3), $n$ stands for the dimension of the covariate, and the covariate $\lambda^{T}(t)$ has the same dimension as the state variable $x(t)$. The Hamiltonian function is defined as in equation (4).

$$
H(x, u, \lambda, t)=L(x, u, t)+\lambda^{T} \cdot f(x, u, t)
$$

PMP points out that the optimal state trajectory $\mathrm{x}^{*}$, the optimal control $\mathrm{u}^{*}$, and the corresponding Lagrange multiplier $\lambda^{*}$ must minimize the Hamilton equation $H$. As a result, $\forall t \in\left[t_{0}, t_{f}\right]$ and $\forall u \in U$ can fall into the allowable control set, as shown in equation (5).

$$
H\left(x^{*}(t), \lambda^{*}(t), u^{*}(t)\right)<H\left(x^{*}(t), \lambda^{*}(t), u(t)\right)
$$

These are only necessary (insufficient) conditions for an optimal control law. The set of control laws satisfying these conditions is called the extremum of the control problem. If there is an optimum, it is also an extremum. On the contrary, it may be extremum rather than optimum. If the optimal control law is unique, these conditions provide a clear way to find the optimal control law.

When solving the HEV energy management optimization problem, PMP requires all the working conditions, so it cannot be used in real-time applications. Because of fast calculation, the ECMS algorithm can implement optimal control decisions and is widely used in instantaneous optimal energy management algorithms.

\subsection{ECMS based on PMP}

The ECMS is derived from engineering experience. The energy source of power-maintained HEV ultimately all comes from fuel, even if it is driven by battery power [15-17]. Therefore, the fuel consumption during the driving cycle also includes 
the fuel consumption equivalent to the energy used from the battery.

The sum of actual fuel consumption rate and electrical fuel consumption rate is called equivalent fuel consumption rate, and their relationship is explained in equation (6).

$$
\dot{m}_{f, e q v}=\dot{m}_{f}+\dot{m}_{f, e l e c}=\dot{m}_{f}+s \frac{p_{\text {batt }}}{Q_{L H V}}
$$

In equation (6), $\dot{m}_{f, e q v}$ denotes the equivalent fuel rate, $s(\mathrm{t})$ represents the equivalent factor between power and fuel, and $Q_{L H V}$ stands for the average low calorific value of the fuel. The minimum equivalent fuel consumption $\left(\dot{m}_{f, e q v}\right)$ in the driving cycle is calculated to solve the energy management problem. The global optimization problem in the whole driving cycle is transformed into solving the local minimization problem of minimizing the equivalent fuel consumption at every moment. In the expression of ECMS, the equivalent fuel consumption rate is expressed by the equivalent factor $s(\mathrm{t})$, battery power $p_{b a t t}$, and fuel calorific value $Q_{L H V}$. Because the conversion efficiency between battery energy and fuel is not included in equation (6), the loss involved is included in the equivalent factor $s(\mathrm{t})$. Here, the same equivalent factor $(s)$ is used for both battery charging and battery discharging. To reduce the number of calibration parameters, the equivalent fuel consumption rate can be expressed as in equation (7).

$$
\dot{m}_{f, e q v}=\dot{m}_{f}+\dot{m}_{f, e l e c}=\dot{m}_{f}+s \frac{p_{\text {batt chem }}}{Q_{L H V}}
$$

In equation (7), $p_{\text {batt, }}$ chem represents the interpolated chemical battery power (including battery efficiency). The product of $\mathrm{V}_{\text {oc.max }}$ and battery current I represents the battery power $p_{\text {batt, }}$ chem.

\subsection{Energy Management based on WL-ECMS (ECMS based on the Willans Line Model)}

First, the basic principle of ECMS is introduced for the energy optimization problem of HEVs without external charging. Since the SOC of the power battery has little influence on the parameters, such as voltage and internal resistance, the equivalent factor of ECMS can be approximately equalled to the co-state of PMP. Thus, the ECMS optimization problem can be approximately equalled to the PMP optimization problem $[18,19]$. The essence of the ECMS optimization problem is to convert battery energy consumption into equivalent fuel consumption through equivalent factors. Subsequently, the power of the battery is taken as the control variable. Before the ECMS optimization, the feasible region of the battery power is discretized, and then the corresponding engine output power is obtained according to the vehicle power balance equation. In the ECMS optimization process, the equivalent fuel consumptions corresponding to the discrete grid points of the engine and the power battery are compared, and then the optimal fuel economy is obtained. Finally, the control variable corresponding to the optimal fuel economy is returned, which is the optimal control variable [20]. ECMS optimization problem is equivalent to interpolation and look-up table problem, that is, to find the optimum in the table and the corresponding control variables. Compared with global optimization algorithms, such as dynamic programming algorithms and GA (Genetic Algorithms), ECMS can significantly reduce the calculation complexity without sacrificing the accuracy of the optimum. Therefore, ECMS is one of the most promising control algorithms for real vehicle controllers. However, compared with the data calculation ability of the current vehicle processor, the calculation complexity of the search method for obtaining time-varying discrete tables, interpolation, lookup tables, and re-optimization is still too high. Moreover, with higher demand for calculation accuracy, the number of discrete points needs to be increased. This, however, is bound to increase the dimension of time-varying tables, and at the same time, the calculation time will also be increased, which is why ECMS cannot realize online optimization based on real vehicle controllers [2124].

To apply ECMS to real vehicle controllers, some researchers have proposed an off-line simulation method to obtain the optimal control set table and store it in the vehicle controller. Compared with the on-line ECMS, this method does not calculate the time-varying table. However, the optimal driving performance of the vehicle cannot be ensured through the off-line ECMS [25]. The reason is that the MAP (Manifold Absolute Pressure) diagrams of the engine and ISG (Integrated Starter and Generator) motor are irregular, so the obtained ECMS table will have mutations. This will inevitably lead to the state mutation of the engine, thus affecting the driving performance. Some scholars have proposed a method based on local search to reduce the calculation of ECMS, but this method is still too large. Some scholars have proposed a simplified power component model to reduce the calculation complexity of ECMS. Among them, ECMS based on the Willans Line model of engine and motor has been widely concerned because of its superior performance. This method is called WL-ECMS [26].

Based on the linearization concept, the Willans Line model of the engine can be expressed as in equation (8).

$$
P_{e f}=a_{e} P_{e m}+b_{e}
$$

In equation (8), $P_{\text {ef }}$ represents the power emission of the fuel during engine combustion, $P_{e m}$ 
denotes the effective power of the engine, and $a_{e}$ stands for the reciprocal of the indicated efficiency of the engine. $b_{e}$ denotes the regression coefficient of engine power loss. Meanwhile, both $\mathrm{a}_{e}$ and $\mathrm{b}_{e}$ are functions related to engine speed.

Similarly, based on the linearization idea, the Willans Line model of the ISG motor can be expressed as in equation (9).

$$
P_{m e}= \begin{cases}a_{m 1} p_{m m}+b_{m 1} & p_{m n}>0 \\ 0 & p_{m n}=0 \\ a_{m 1} p_{m m}+b_{m 1} & p_{m n}<0\end{cases}
$$

In equation (9), $P_{m e}$ denotes the output power of the battery, and its value is equal to the effective power of ISG motor output divided by the corresponding system efficiency. $P_{m m}$ represents the effective output power of the motor. Parameters $a_{m 1}, b_{m 1}, a_{m 2}$, and $b_{m 2}$ related to ISG motor speed are regression coefficients.

The form of the ECMS optimization problem is consistent with the Willans Line model of power components. Based on the equivalent voltage and current, the simplified ECMS optimization problem can be expressed as in equation (10).

$$
\left\{\begin{array}{c}
p_{e q}=p_{e f}-\mu I_{b a t} \\
\mu=\lambda H_{L H V}
\end{array}\right.
$$

In equation (10), $p_{e q}$ denotes the equivalent power of ECMS, $\mu$ stands for the equivalent voltage related to the equivalent power corresponding to the unit current of the battery, and $\lambda$ represents the equivalent factor of WL-ECMS.

\subsection{Energy Management based on A-ECMS}

The effect of an equivalent factor on the optimization of the ECMS energy management algorithm is very obvious. The ECMS algorithm with constant equivalent factor has poor adaptability in actual working conditions, which cannot effectively improve the fuel economy of HEVs. This is also the challenge of the ECMS algorithm in practical application [27,28]. To solve the above problems, relevant researchers have proposed an AdaptiveECMS, or A-ECMS (Adaptive ECMS), algorithm.

The basic principle is to self-adjust the equivalent factor based on relevant information in the driving process and improves the applicability of ECMS [29].

A stable battery SOC is a crucial problem in AECMS applications to prevent battery overcharge or over-discharge. The control process is shown in figure 1.

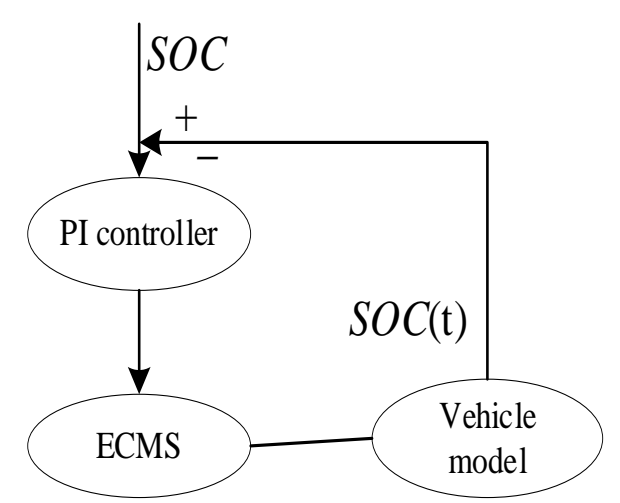

Figure1 Principle of A-ECMS algorithm

In essence, the A-ECMS control algorithm is an ECMS with closed-loop feedback control of equivalent factors. Since the ECMS adaptation is to ensure the charge retention operation, the feedback will come from the SOC of the battery. Here, two adaptive methods are considered: feedback at each time point (continuous) and feedback only at the discrete-time (discrete). Similar to ECMS, only a single equivalent factor ( $\mathrm{s}$ ) is used for charging and discharging in these two methods. Studies have shown that the result of usage of a single equivalent factor is only slightly better than that of multiple equivalent factors (charging and discharging) [30].

The summary of the A-ECMS control algorithm is based on the following facts. (1) The optimum of equivalent factor is the optimum of power maintenance. (2) If the equivalent factor is less than the optimum, the battery SOC tends to decrease, and if the equivalent factor is too high, the battery SOC tends to increase. (3) The ideal required interval of charge sustainability should be the entire journey, but this is impossible because its duration is unknown. Therefore, it is assumed that SOC should return to the reference value at a fixed interval of duration. (4) The charge sustainability interval $\mathrm{T}$ should be long enough to allow the battery to charge and discharge in the whole working range. Based on these facts, the adaptive policy updates the value of the equivalent factor at each end of these intervals (defined as adaptive intervals).

The basic steps to achieve efficient feedback are as follows. (1) In the first step of the simulation, the speed curve is optimized through the initial value of SMPC utilization efficiency. (2) After the current speed and the actual output speed of the vehicle is obtained, the required torque and power of the vehicle are calculated based on the PID driver model. (3) The transmission ratio and efficiency of the automatic transmission are calculated based on the main parameters, such as vehicle demand torque, power, and vehicle speed. 
(4) The output parameters of the engine and ISG motor can be obtained through the required torque, power, and vehicle control algorithm, namely, the automatic transmission speed ratio. Based on this, driving and energy recovery efficiency can be calculated. (5) The calculated efficiency is fed back to the fuel consumption model of the upper controller, and the optimal target vehicle speed at the next moment is optimized according to the updated fuel consumption model. Then, the procedure will be repeated back and forth.

At present, almost all the control methods based on optimization have a common problem, that is, to achieve online optimization, the known cycle conditions are needed, or the known cycle conditions can improve the performance of the control algorithm [31]. For example, in DP (Dynamic Programming) method, the premise of global optimization is that the whole cycle condition is known, which is difficult to achieve in traditional HEV energy management. In ECMS, if the cycle conditions are known, the optimal equivalent factor can be obtained through optimization, and consequently, a better fuel economy can be gained together with a balanced SOC power battery. At present, there are many works of academic literature about the acquisition methods of cycle conditions, such as the condition prediction based on Markov decision, the condition identification based on neural network, and the condition prediction based on GPS (Global Positioning System) or GIS (Geographic Information System). These methods have some drawbacks, including poor real-time performance and poor accuracy. Besides, their optimization models are limited to specific conditions. To overcome these problems, some scholars have studied the speed prediction method based on the intelligent transportation system and used MPC (Model Predictive Control) algorithm to obtain the optimal target speed, including the speed optimization of a single vehicle and the speed optimization of a fleet containing multiple vehicles. Yet, there is little literature on the optimization of ECMS equivalent factors of multiple HEVs based on the speed predicted by intelligent network connection MPC, and there is little literature on the global optimization of energy management of multiple HEVs based on the predicted working conditions [32].

A properly-designed ECMS control algorithm can well maintain the SOC balance of the power battery. A fixed equivalent factor is chosen in the ECMS. When other driving conditions are replaced, the equivalent factor also needs to be adjusted. Otherwise, the control effect of ECMS may become worse. Therefore, some scholars have proposed to use A-ECMS for the energy management of HEVs. Compared with ECMS, A-ECMS has better adaptability to working conditions and can show good control effects in most working conditions [33].
Nevertheless, to ensure good performance under different working conditions, the final SOC of different vehicles varies tremendously. This indicates that when the working conditions change greatly, the A-ECMS method may not be able to balance the charge. The parameters of the A-ECMS control algorithm also need to be properly adjusted for different operating conditions [34,35]. Before the ECMS parameters can be adjusted, the cycle conditions should be known, and there are few works of literature on the optimization of equivalent factors through the prediction speed based on the intelligent network connection. Here, the prediction speed information can optimize the equivalent factor. The energy management diagram of equivalent factor optimization based on prediction information is shown in figure 2 .

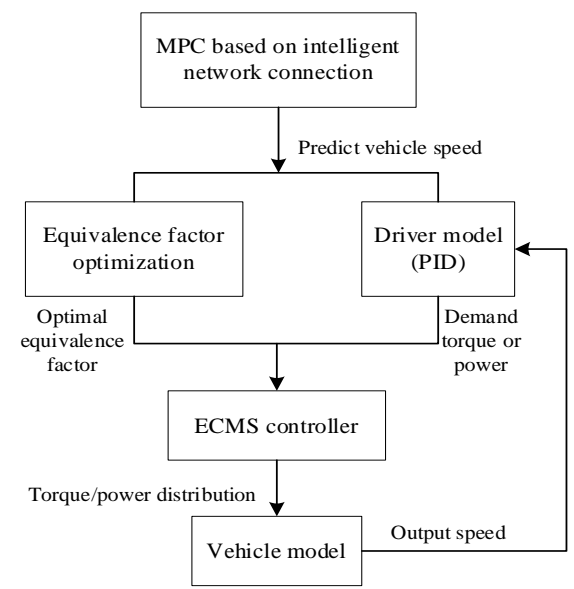

Figure 2 Energy management flowchart of equivalent factor optimization based on prediction information

ECMS equivalent factor optimization is a nonlinear, non-differentiable, and constrained optimization problem. The GA can also optimize this kind of problem. Through a probabilistic search mechanism, GA can search the global optimum of multimodal optimization problems, as shown in figure 3. The optimization principle of GA is to evaluate the fitness of the population adaptive function value and then select, crossover, mutate to determine the next generation of the population. Finally, stop iteration until the stop criterion is met [36].

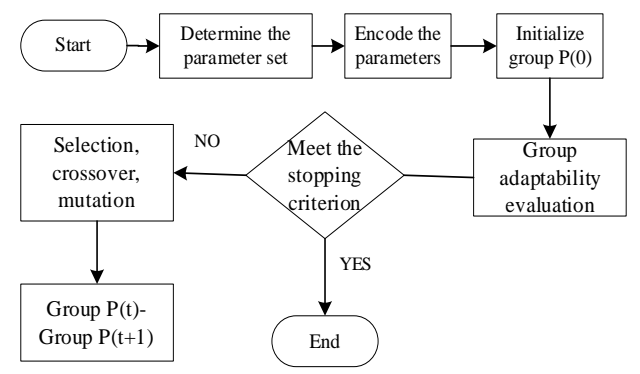

Figure 3 Optimization principle of GA 


\section{Simulation Results \\ 3.1 ECMS Simulation Results after Equivalent Optimization}

The trajectory curves of four vehicles are obtained through MATLAB simulation based on UDP (User Datagram Protocol) communication, as shown in figure 4.

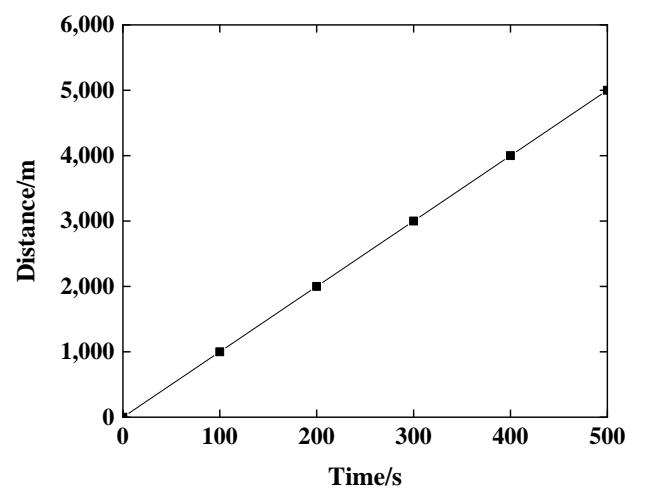

Figure 4 Vehicle speed trajectory based on the intelligent network connection

Figure 4 suggests that four four-wheel-drive HEVs pass through 13 signal lights continuously, and their trajectories do not intersect with the red-light time window, so no vehicle has stopped during the red light. Besides, the trajectory of each vehicle has no intersection point, indicating that the intelligent network connection method can avoid the collision of four-wheel-drive HEVs.

\subsection{Control Effect of Real-time Efficiency Feedback}

To visually verify the impact of efficiency feedback on fuel economy, the fuel economy comparison of vehicles is illustrated with and without efficiency feedback, as shown in figure 5 .

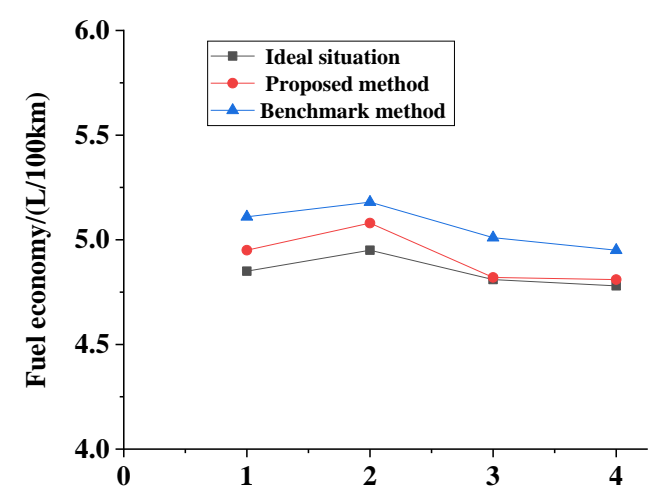

(a) Vehicle serial number

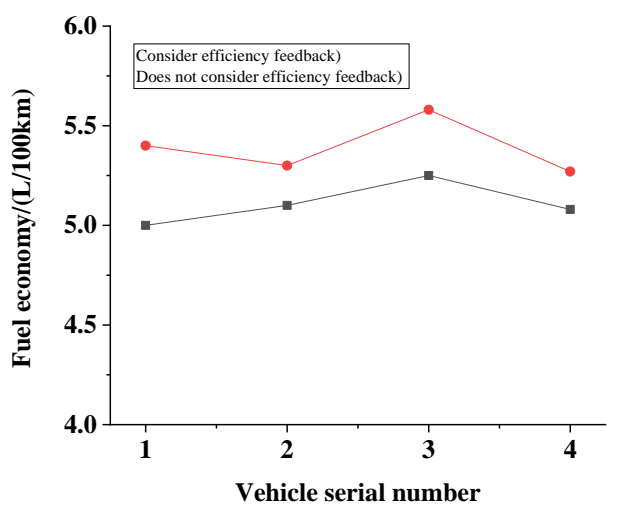

Figure 5 Fuel economy feedback results

Figure 5 implies that the fuel economy of each vehicle with real-time efficiency feedback is higher than that without efficiency feedback. The average fuel economy of the four vehicles can be calculated. The fuel consumption per $100 \mathrm{~km}$ with efficiency feedback is $5.06 \mathrm{~L}$, which is $6.47 \%$ higher than 5.41 $\mathrm{L} / 100 \mathrm{~km}$ of that without efficiency feedback.

The speed curve indicates that the closed-loop hierarchical control model is established through real-time efficiency feedback. The fuel consumption model in the upper control system is corrected in real-time and becomes more accurate. Therefore, a smoother speed curve can be obtained through an optimized fuel consumption model which includes the real-time efficiency feedback. With a smooth speed, the HEV can work more efficiently, obtaining a higher fuel economy.

The shape of the optimal speed curve of SPAT (Node Levelling Network Adjustment Calculation) is of great significance. To verify the control effect of the proposed control algorithm under other SPATs, the fuel economy of upper and lower control methods of SPAT3015 are illustrated for comparison, as shown in figure 6.

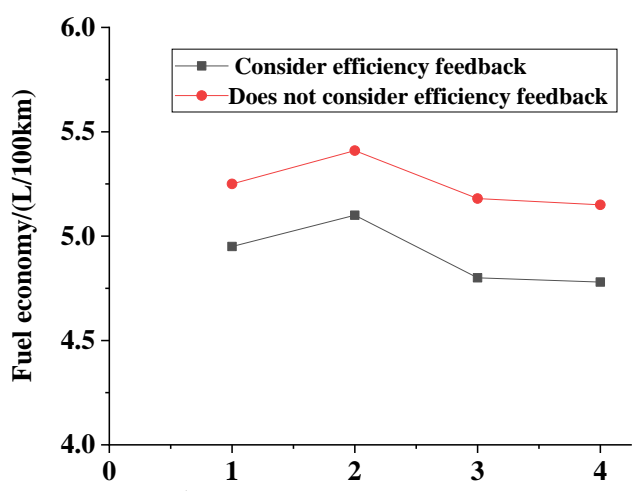

(b) Vehicle serial number

Figure 6 Results of fuel economy comparison (a) Fuel economy comparison under different methods (b) Fuel economy comparison under efficiency feedback

Figure 6 (a) indicates that the fuel economy of each HEV based on ECMS is closer to the ideal situation than the benchmark method under SPAT3015. The average fuel consumption is $4.95 \mathrm{~L}$ 
based on ECMS and 5.07 L based on the benchmark method.

The fuel economy of the ECMS method is $2.37 \%$ higher than that of the benchmark method. Figure 6 (b) shows the comparison of $100 \mathrm{~km}$ fuel consumption considering efficiency feedback or not.

Obviously, for any vehicle, the fuel economy considering efficiency feedback is higher than that without efficiency feedback. For the average fuel consumption of $100 \mathrm{~km}$, the average fuel economy is $4.95 \mathrm{~L}$ with efficiency feedback and $5.22 \mathrm{~L}$ without efficiency feedback.

The average fuel economy increases by $5.17 \%$ with efficiency feedback.

\subsection{Energy Control Effect of A-ECMS based on SOC Feedback}

Compared with the traditional ECMS, dA-ECMS (Equivalent Factor Adaptive Control Strategy Based on Discrete SOC Feedback) and cA-ECMS (Equivalent Factor Adaptive Control Strategy Based on Continuous SOC Feedback) are adaptive improvements based on the control principle of traditional ECMS. Their influence on fuel consumption reduction is more intuitive than ECMS. Figure 7 shows the extent to which dA-ECMS and cAECMS deviate from the reference value at the end of the operation at different initial SOC values

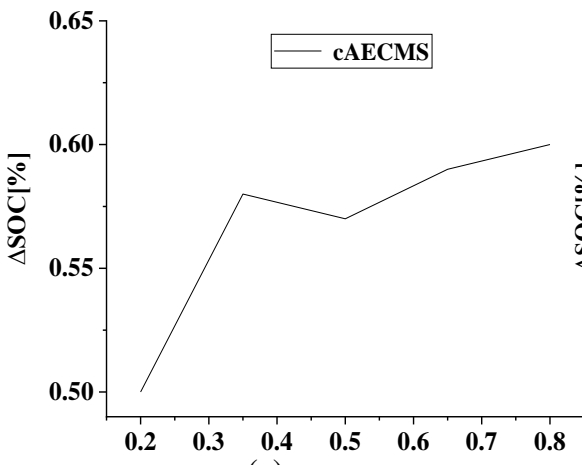

(a) SoCinit

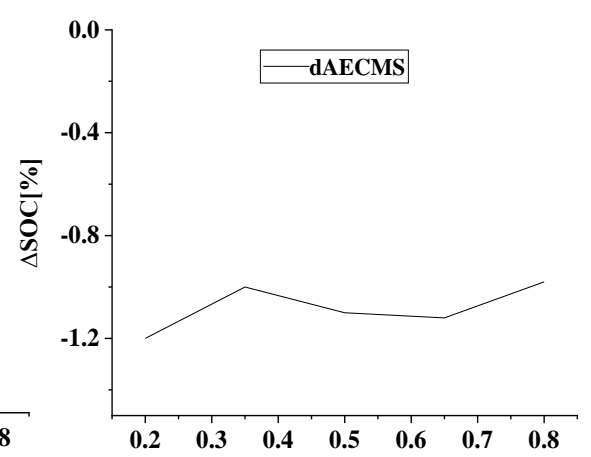

(b) SOCinit

Figure 7 A-ECMS battery end SOC deviation: (a) cA-ECMS; (b) dA-ECMS

Figure 7 shows that the dA-ECMS control algorithm based on discrete SOC feedback and the cA-ECMS control algorithm based on continuous SOC feedback has little difference in the equivalent fuel consumption and SOC curve, and the fuel-saving effect is similar. There are some differences between different working conditions. The traditional ECMS should obtain the road conditions in advance and obtain the optimal $\mathrm{S}_{0}$ through PMP, so under the same circumstances, the fuel economy is slightly better than the A-ECMS control algorithm based on SOC feedback. While the real-time performance of AECMS is significantly better than that of the traditional ECMS [37].

The transition from traditional ECMS to dA-ECMS and cA-ECMS is shown in figure 8.

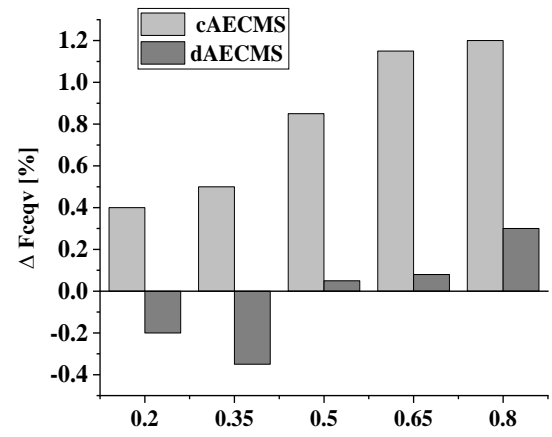

(a) SoCinit

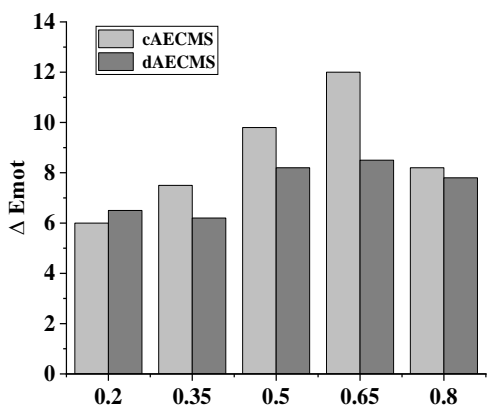

(b) SOC init

Figure 8 The change degree of ECMS to CA-ECMS and AA-ECMS (a) The change rate of equivalent fuel consumption from ECMS to A-ECMS; (b) The change rate in battery usage from ECMS to A-ECMS

Figure 8 suggests that the overall trend of equivalent fuel consumption of dA-ECMS and cAECMS gradually decreases with the increase of SOC initial value.
While, compared with the traditional ECMS, the oil-saving rate decreased first and then increased, with a maximum increase of $1.1486 \%$ and a maximum decrease of $0.3465 \%$. 
In some cases, dA-ECMS and cA-ECMS consume less fuel than traditional ECMS, because their SOC initial value is lower than the SOC reference value of traditional ECMS. Although the goal of dA-ECMS and cA-ECMS adaptive control strategies is to ensure long-term power maintenance, the ultimate value of SOC does not have to equal to the initial value, so a slight gap is acceptable in real vehicle scenarios. As expected, the energy consumption of the battery has the same trend as the fuel consumption. Compared with traditional ECMS, the battery consumption of the cA-ECMS control algorithm based on continuous SOC feedback is the highest, while the battery consumption of dA-ECMS based on discrete SOC feedback is the second highest.

\section{Conclusion}

Here, the energy optimization management control method is studied of the four-wheel-drive HEV with predictive information. According to the rule-based control algorithm, the control algorithm based on ECMS is designed. Then, cA-ECMS and dAECMS are proposed. Combined with the A-ECMS control algorithm, an online identification A-ECMS algorithm utilizing SOC feedback is established. The results show that the control algorithm gives full play to the advantages of the adaptive control algorithm and improves the adaptability of equivalent factors of HEVs. The equivalent fuel consumption and fuel-saving are over $15 \%$. Besides, the fuel consumption, the total energy consumption of the motor, and the deviation of the SOC end value under different SOC initial values are compared. The results show that with the increase of the SOC initial value, the SOC deviation of the end state of the cAECMS and dA-ECMS control algorithms are about $0.6 \%$ and $-1.6 \%$, respectively. Meanwhile, the fuel consumption increases continuously, and the cAECMS control algorithm tends to use fewer batteries. From the perspective of the adaptability of the equivalent factor, the equivalent factor of the $\mathrm{dA}$ ECMS control algorithm changes slightly and has good adaptability. This lays a solid theoretical foundation for the establishment of an energy management control algorithm for real-time vehicle control.

\section{Acknowledgment}

This research is supported by Jiangsu Higher Vocational College Teachers' professional leader high-end training Project (2019GRFX019), Changzhou Key Laboratory of high technology (CM20183004) and the Project of "1+1+1" Cooperative Nurturing Engineering Construction in Changzhou College of Information Technology (Grant No: XTPY201706).

\section{References}

[1] Wang, Y., Wang, X., Sun, Y. (2018) Model predictive control strategy for energy optimization of series-parallel hybrid electric vehicle. Journal of cleaner production, 199(6), 348-358.

[2] Sulaiman N., Hannan M. A., Daud, W. W. (2018) Optimization of energy management system for fuel-cell hybrid electric vehicles: Issues and recommendations. Applied energy, 228(15), 2061-2079.

[3] Guo H., Wang X., Li L. (2019) State-of-chargeconstraint-based energy management strategy of plug-in hybrid electric vehicle with bus route. Energy Conversion and Management, 199(13), 111972.

[4] Zulkefli M. A. M., Sun Z. (2019) Fast numerical powertrain optimization strategy for connected hybrid electric vehicles. IEEE Transactions on Vehicular Technology, 68(9), 8629-8641.

[5] Zeng Y., Cai Y., Qin D. (2018) Energy management for plug-in hybrid electric vehicle based on adaptive simplified-ECMS. Sustainability, 10(6), 2060.

[6] Rezaei A., Burl J. B., Zhou B. (2017). Estimation of the ECMS equivalent factor bounds for hybrid electric vehicles. IEEE Transactions on Control Systems Technology, 26(6), 2198-2205.

[7] Veerendra A. S., Mohamed M. R., Leung P. K. (2021) Hybrid power management for fuel cell/supercapacitor series hybrid electric vehicle. International Journal of Green Energy, 18(2), 128-143.

[8] Yue M., Jemei S., Zerhouni N. (2019) Review on health-conscious energy management strategies for fuel cell hybrid electric vehicles: Degradation models and strategies. International Journal of Hydrogen Energy, 44(13), 6844-6861.

[9] Noura N., Boulon L., Jemeï S. (2020) A Review of Battery State of Health Estimation Methods: Hybrid Electric Vehicle Challenges. World Electric Vehicle Journal, 11(4), 66.

[10] Prajwowski K., Golebiewski W., Galdynski, D. (2020) Modeling of working machines synergy in the process of the hybrid electric vehicle acceleration. Energies, 13(21), 5818.

[11] Das H. S., Tan C. W., Yatim A. H. M. (2017) Fuel cell hybrid electric vehicles: A review on power conditioning units and topologies. Renewable and Sustainable Energy Reviews, 76(9), 268-291.

[12] Huang Y., Wang H., Ji J. (2017) Model predictive control power management strategies for HEVs: A review. Journal of Power Sources, 341(11), 91106.

[13] Shi D., Wang S., Chen L. (2018) Stochastic predictive energy management of power split hybrid electric bus for real-world driving cycles. IEEE Access, 6(2), 61700-61713. 
[14] Ma H., Simon D., Fei M. (2017) Biogeographybased optimization: a 10-year review. IEEE Transactions on Emerging Topics in Computational Intelligence, 1(5), 391-407.

[15] Uebel S., Murgovski N., Sjöberg, J. (2019) A twolevel mpc for energy management including velocity control of hybrid electric vehicles. IEEE Transactions on Vehicular Technology, 68(6), 5494-5505.

[16] Dissanayake A. M., Ekneligoda N. C. (2018). Transient optimization of parallel connected inverters in islanded ac microgrids. IEEE Transactions on Smart Grid, 10(5), 4951-4961.

[17] Shen M., Gao Q. (2019) A review on battery management system from the modeling efforts to its multiapplication and integration. International Journal of Energy Research, 43(10), 5042-5075.

[18] Wang X., Ma S., Wang J. (2018) Predictive energy management for hybrid electric vehicles considering extension of the battery life. Proceedings of the Institution of Mechanical Engineers, Part D: Journal of Automobile Engineering, 232(4), 499-510.

[19] Hua Y., Zhou S., Yang S. (2020) A comprehensive review on inconsistency and equalization technology of lithium-ion battery for electric vehicles. International Journal of Energy Research, 44(14), 11059-11087.

[20] Rizzo G., Naghinajad S., Marino M. (2020) A Survey on Through-the-Road Hybrid Electric Vehicles. Electronics, 9(5), 879.

[21] Yang L., Cai Y., Deng Z. (2020) Supervisory longterm prediction of state of available power for lithium-ion batteries in electric vehicles. Applied Energy, 257(21), 114006.

[22] Hu X., Jiang H., Liu B. (2020) An enhanced multistate estimation hierarchy for advanced lithiumion battery management. Applied Energy, 257(13), 114019.

[23] Xu X., Lin Y., Zhou Z. (2019) A hybrid observer for SOC estimation of lithium-ion battery based on a coupled electrochemical-thermal model. International Journal of Green Energy, 16(15), 1527-1538.

[24] Ouyang T., Xu P., Chen N. (2020) Improved parameters identification and state of charge estimation for lithium-ion battery with real-time optimal forgetting factor. Electrochimica Acta, 353(8), 136576.

[25] Stroe N., Olaru S., Chamaillard Y. (2019) Predictive Control Framework for HEV: Energy Management and Free-Wheeling Analysis. IEEE Transactions on Intelligent Vehicles, 4(2), 220231.
[26] Lin C., Tang A., Xing J. (2017) Evaluation of electrochemical models based battery state-ofcharge estimation approaches for electric vehicles. Applied Energy, 207(11), 394-404.

[27] Tudoroiu N., Zaheeruddin M., Tudoroiu R. E. (2020) Real Time Design and Implementation of State of Charge Estimators for a Rechargeable Lithium-Ion Cobalt Battery with Applicability in HEVs/EVs-A Comparative Study. Energies, 13(11), 2749.

[28] Li W., Yang Y., Yin S. (2020) The multiinnovation extended Kalman filter algorithm for battery SOC estimation. Ionics, 26(12), 61456156.

[29] Hu X., Cao D., Egardt B. (2017) Condition monitoring in advanced battery management systems: Moving horizon estimation using a reduced electrochemical model. IEEE/ASME Transactions on Mechatronics, 23(1), 167-178.

[30] Xia B., Chen G., Wang H. (2019) Online Parameter Identification and Joint Estimation of the State of Charge and the State of Health of Lithium-Ion Batteries Considering the Degree of Polarization. Energies, 12(15), 2939.

[31] Guanetti J., Formentin S., Savaresi S. M. (2017) Optimal energy management in series hybrid electric bicycles. Automatica, 81(5), 96-106.

[32] Xiang Y., Yang X. (2021) An ECMS for MultiObjective Energy Management Strategy of Parallel Diesel Electric Hybrid Ship Based on Ant Colony Optimization Algorithm. Energies, 14(4), 810.

[33] Xia B., Zheng W., Sun Z. (2017) A novel observer for lithium-ion battery state of charge estimation in electric vehicles based on a second-order equivalent circuit model. Energies, 10(8), 1150.

[34] Fang Q., Wei X., Dai H. (2019) A remaining discharge energy prediction method for lithiumion battery pack considering SOC and parameter inconsistency. Energies, 12(6), 987.

[35] Shin D., Yoon B., Yoo S. (2021) Compensation Method for Estimating the State of Charge of LiPolymer Batteries Using Multiple Long ShortTerm Memory Networks Based on the Extended Kalman Filter. Energies, 14(2), 349.

[36] Wang S. L., Fernandez C., Zhang L. (2019) A comprehensive working state monitoring method for power battery packs considering state of balance and aging correction. Energy, 171, 444455.

[37] Plett G. L. (2019) Review and some perspectives on different methods to estimate state of charge of lithium-ion batteries. Journal of Automotive Safety and Energy, 10(3), 249. 